\title{
Determining All Universal Tilers
}

\author{
David G.L. Wang
}

Received: 12 February 2012 / Revised: 2 July 2012 / Accepted: 5 July 2012 /

Published online: 21 July 2012

(C) Springer Science+Business Media, LLC 2012

\begin{abstract}
A universal tiler is a convex polyhedron whose every cross-section tiles the plane. In this paper, we introduce a slight-rotating operation for cross-sections of polyhedra. By applying the operation to suitably chosen cross-sections, we prove that a convex polyhedron is a universal tiler if and only if it is a tetrahedron or a pentahedron with parallel facets.
\end{abstract}

Keywords Cross-section · The slight-rotating operation · Universal tiler

\section{Introduction}

A tiler is a polygon that can cover the plane by congruent repetitions without gaps or overlaps. The problem of determining all tilers, which is alternatively called the problem of tessellation or of plane tiling, is one of the most famous problems in discrete mathematics. It is still open to the best of our knowledge. The reader is referred to Grunbaum and Shephard's book [3] for an introduction to the theory of tessellation, and to Brass, Moser and Pach's book [2] for many further problems and results on tiling.

Considering a variant of the problem of plane tiling, Akiyama [1] found all convex polyhedra whose every development is a tiler. The key idea in his proof is to find whether the facets of a polyhedron tile the plane in a certain stamping manner. Noticing that facets are special cross-sections, we study another variant of the problem of plane tiling, namely, what kind of polyhedra are so well performed that each of its cross-sections is a tiler.

D.G.L. Wang $(\bowtie)$

Beijing International Center for Mathematical Research, Peking University, Beijing 100871, P.R. China

e-mail:wg1@math.pku.edu.cn 
Let $\mathcal{P}$ be a convex polyhedron, and $\pi$ a plane. Denote the intersection of $\pi$ and $\mathcal{P}$ by $C(\pi)$. We say that $\pi$ intersects $\mathcal{P}$ non-trivially if $C(\pi)$ is a non-degenerated polygon, that is, $C(\pi)$ is a polygon with at least 3 edges. We call $C(\pi)$ a cross-section if $\pi$ crosses $\mathcal{P}$ nontrivially. The polyhedron $\mathcal{P}$ is said to be a universal tiler if every cross-section of $\mathcal{P}$ is a tiler. The goal of this paper is to determine all universal tilers.

With the aid of Euler's formula, and Reinhardt's theorem [5], the author [7] managed to obtain the following necessary condition for the number of faces of a universal tiler by investigating specific cross-sections of any given polyhedron.

Theorem 1.1 Any universal tiler has at most five facets. Moreover, a convex polyhedron is a universal tiler if it is a tetrahedron or a pentahedron with parallel facets.

In light of the above theorem, the problem of determining all universal tilers turns out to be one of finding the list of pentahedronal universal tilers. One of the difficulties in determining whether a pentahedron is a universal tiler is the fact that the list of pentagonal tilers is not known, although there are 14 classes of pentagonal tilers are found, see Hirschhorn and Hunt [4], and Sugimoto and Ogawa [6] for instance.

The key idea used in solving the universal tiler problem consists of two parts. The first part is to construct some technical conditions subject to which we can specify a cross-section of any given pentahedron. It is an extension of the method adopted in [7]. The other part is to introduce a slight-rotating operation and apply it to the specified cross-sections. Here is our main result.

Theorem 1.2 A convex polyhedron is a universal tiler if and only if it is a tetrahedron or a pentahedron with parallel facets.

Our proof of the above theorem has nothing to do with the knowledge of the complete list of pentagonal tilers. This paper is organized as follows. In the next section, we give necessary notions and notations on tessellations of the plane formed by a single pentagon. Section 3 is devoted to the construction of technical conditions and to the introduction of the slight-rotating operation. In Sect. 4, we complete the proof of Theorem 1.2.

\section{Preliminary}

In this section, we introduce some necessary notions and notations. Let $T=$ $V^{1} V^{2} \cdots V^{5}$ be a pentagonal tiler. Let $\mathcal{T}$ be a tessellation of the plane by copies of $T$. Denote the copies used in $\mathcal{T}$ by $\left\{T_{i}=V_{i}^{1} V_{i}^{2} \cdots V_{i}^{5}: i \in \Lambda\right\}$, where $\Lambda$ is a set. Then every $T_{i}$ has the same shape as $T$.

Let $i \in \Lambda$ and $\varepsilon>0$. Since $T$ is a tiler, the $\varepsilon$-neighborhood of the point $V_{i}^{j}$ in $\mathcal{T}$ must be covered without gaps or overlaps. It follows that either there is a sequence $V_{i}^{j}, V_{i_{1}}^{j_{1}}, V_{i_{2}}^{j_{2}}, \ldots, V_{i_{k}}^{j_{k}}(k \geq 2)$ of angles arranged counter-clockwise which complete the $2 \pi$ angle around the point $V_{i}^{j}$, or there is a sequence $V_{i_{1}}^{j_{1}}, \ldots, V_{i_{k}}^{j_{k}}, V_{i}^{j}, V_{s_{1}}^{t_{1}}, \ldots$, 
Fig. 1 The local tessellation around the point $V_{i}^{j}$
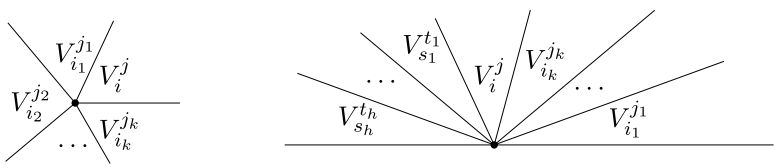

Fig. 2 The arrangement (2.3)

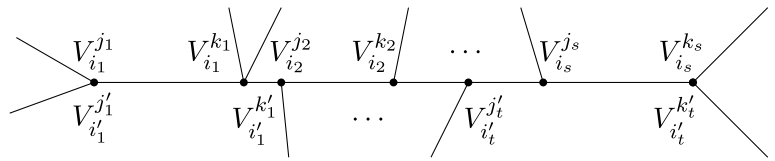

$V_{s_{h}}^{t_{h}}(k+h \geq 1)$ of angles arranged counter-clockwise which adds to $\pi$ (see Fig. 1). In the former case, we denote the local tessellation around the point $V_{i}^{j}$ by

$$
S\left(V_{i}^{j}\right)=\left[V_{i}^{j}, V_{i_{1}}^{j_{1}}, V_{i_{2}}^{j_{2}}, \ldots, V_{i_{k}}^{j_{k}}\right] .
$$

In the latter case, we denote it by

$$
S\left(V_{i}^{j}\right)=\left[V_{i}^{j}, V_{s_{1}}^{t_{1}}, \ldots, V_{s_{h}}^{t_{h}}, \pi, V_{i_{1}}^{j_{1}}, \ldots, V_{i_{k}}^{j_{k}}\right] .
$$

Suppose that there is a sequence $V_{i_{1}}^{j_{1}} V_{i_{1}}^{k_{1}}, V_{i_{2}}^{j_{2}} V_{i_{2}}^{k_{2}}, \ldots, V_{i_{s}}^{j_{s}} V_{i_{s}}^{k_{s}}$ of $s$ collinear edges such that the point $V_{i_{r}}^{k_{r}}$ coincides with the point $V_{i_{r+1}}^{j_{r+1}}$ for each $1 \leq r \leq s-1$, and the copies $T_{i_{1}}, T_{i_{2}}, \ldots, T_{i_{s}}$ lie on the same side of the directed line segment $\vec{l}=V_{i_{1}}^{j_{1}} V_{i_{s}}^{k_{s}}$. In this case, we denote

$$
\vec{l}=V_{i_{1}}^{j_{1}} V_{i_{1}}^{k_{1}}+V_{i_{2}}^{j_{2}} V_{i_{2}}^{k_{2}}+\cdots+V_{i_{s}}^{j_{s}} V_{i_{s}}^{k_{s}} .
$$

Assume also that

$$
\vec{l}=V_{i_{1}^{\prime}}^{j_{1}^{\prime}} V_{i_{1}^{\prime}}^{k_{1}^{\prime}}+V_{i_{2}^{\prime}}^{j_{2}^{\prime}} V_{i_{2}^{\prime}}^{k_{2}^{\prime}}+\cdots+V_{i_{t}^{\prime}}^{j_{t}^{\prime}} V_{i_{t}^{\prime}}^{k_{t}^{\prime}}
$$

such that the copies $T_{i_{1}^{\prime}}, T_{i_{2}^{\prime}}, \ldots, T_{i_{t}^{\prime}}$ lie on the other side of $\vec{l}$ (see Fig. 2). In this case, we say that $\vec{l}$ is represented in $\mathcal{T}$, and is denoted as

$$
S\left(V_{i_{1}}^{j_{1}} V_{i_{1}}^{k_{1}}, V_{i_{2}}^{j_{2}} V_{i_{2}}^{k_{2}}, \ldots, V_{i_{s}}^{j_{s}} V_{i_{s}}^{k_{s}}\right)=\left[V_{i_{1}^{\prime}}^{j_{1}^{\prime}} V_{i_{1}^{\prime}}^{k_{1}^{\prime}}, V_{i_{2}^{\prime}}^{j_{2}^{\prime}} V_{i_{2}^{\prime}}^{k_{2}^{\prime}}, \ldots, V_{i_{t}^{\prime}}^{j_{t}^{\prime}} V_{i_{t}^{\prime}}^{k_{t}^{\prime}}\right]
$$

Let $\mathbb{Z}, \mathbb{Z}^{+}$and $\mathbb{N}$ denote the set of integers, positive integers and nonnegative integers, respectively. Let $N>0$ and $a_{1}, \ldots, a_{5} \in \mathbb{N}$. We say that the set

$$
\left\{a_{1} V^{1}, a_{2} V^{2}, \ldots, a_{5} V^{5}\right\}
$$

is a sum- $N$-collection if $N=\sum_{i=1}^{5} a_{i} V^{i}$. We call the set (2.4) a sum- $N$-collection of the angle $V^{j}$ if $a_{j} \geq 1$. For convenience, we remove the term $a_{i} V^{i}$ from the collection (2.4) if $a_{i}=0$. Denote by $R_{N}\left(V^{j} ; T\right)$ the set of sum- $N$-collections of $V^{j}$ in $T$. To simplify notation, we write $R_{2 \pi}\left(V^{j} ; T\right)=R\left(V^{j} ; T\right)$. Since $T$ is a tiler, we have

$$
R\left(V^{j} ; T\right) \neq \varnothing
$$


Fig. 3 The pentagon $A E F C D$

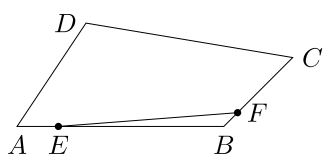

for any $1 \leq j \leq 5$. For example, if

$$
V^{1}=V^{2}=\frac{5 \pi}{6}, \quad V^{3}=\frac{2 \pi}{3}, \quad V^{4}=V^{5}=\frac{\pi}{3},
$$

then we have

$$
\begin{aligned}
R\left(V^{1} ; T\right) & =\left\{\left\{2 V^{1}, V^{4}\right\},\left\{2 V^{1}, V^{5}\right\},\left\{V^{1}, V^{2}, V^{4}\right\},\left\{V^{1}, V^{2}, V^{5}\right\}\right\}, \\
R_{\pi}\left(V^{3} ; T\right) & =\left\{\left\{V^{3}, V^{4}\right\},\left\{V^{3}, V^{5}\right\}\right\} .
\end{aligned}
$$

Note that the sum of any four angles of a pentagon is larger than $2 \pi$, while the sum of any three angles is larger than $\pi$. This immediately leads to the following lemma.

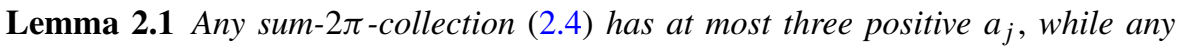
sum- $\pi$-collection (2.4) has at most two positive $a_{j}$.

\section{The Slight-Rotating Operation}

In this section, we construct some technical conditions and introduce the slightrotating operation. As will be seen, for any given pentahedron, there always exists a cross-section satisfying these conditions.

Denote by $\varepsilon$ the set of convex pentahedronal universal tilers without parallel facets. To establish Theorem 1.2, it suffices to prove that $\varepsilon=\varnothing$. We will do this by contradiction. Throughout this paper, we assume that $\mathcal{P} \in \mathcal{E}$.

It is well-known that pentahedra have two distinct topological types. The first consists of quadrilateral-based pyramids. The other typical pentahedra have two triangular bases and three quadrilateral facets joining the corresponding sides of the two bases. In particular, we see that any pentahedron has a quadrilateral facet. Let

$$
Q=A B C D
$$

be a quadrilateral facet of $\mathcal{P}$. Let $E$ be a point lying in the interior of the line segment $A B$ such that $E$ is neither $A$ nor $B$. In this case, we write $E \in A B$. Set another point $F \in B C$. As illustrated in Fig. 3, we have a convex pentagon $T=A E F C D$. In [7], the author took a cross-section of $\mathcal{P}$ by rotating $T$ a bit along the line $E F$, where $E$ is close to $A$, and $F$ is near $B$. In this paper, we extend this method by selecting the points $E$ and $F$ more carefully. The following lemma will be frequently used in the proof of Lemma 3.2.

Lemma 3.1 Let $N \geq 0$. Then the two sets

$$
\left\{\theta>B / 2: N=a \cdot \theta+b \cdot \phi+c \cdot \psi, a \in \mathbb{Z}^{+}, b, c \in \mathbb{N}, \phi, \psi \in\{A, C, D\}\right\}
$$


and

$$
\{l<N: l=a \cdot C D+b \cdot D A+c \cdot A E, a, b \in \mathbb{N}, c \in\{0, \pm 1, \pm 2\}\}
$$

are of finite cardinality.

The above lemma is easy to prove and we omit its proof.

Lemma 3.2 There exists some $\delta_{E}>0$ such that for any point $E \in A B$ with $A E<\delta_{E}$, there exists some $\delta_{F}=\delta_{F}(E)>0$ such that for any point $F \in B C$ with $B F<\delta_{F}$, the pentagon $T=A E F C D$ satisfies

(i) $2 A E<\min \{E F, F C, C D, D A\}$;

(ii) $R(E ; T)=R(F ; T) \subseteq\left\{\{E, F, c \gamma\}: c \in \mathbb{Z}^{+}, \gamma \in\{A, C, D\}\right\}$;

(iii) for any $a, b \in \mathbb{N}, c \in\{0, \pm 1, \pm 2\}$, and $l \in\{E F, 2 F C\}$, we have

$$
l \neq a \cdot C D+b \cdot D A+c \cdot A E .
$$

Proof Choose a point $E \in A B$ and move it towards $A$ such that the point $E$ can be arbitrarily close to but never coincide with the point $A$. Similarly, choose $F \in B C$ and move it towards $B$ such that $F$ can be arbitrarily close to but never coincide with the point $B$. Let

$$
x=\max \{A E, B F\} .
$$

In the above transformation, the pentagon $T$ tends to the quadrilateral $Q$. To be more precise, the lengths of edges vary in such a way that

$$
\lim _{x \rightarrow 0} A E=0, \quad \lim _{x \rightarrow 0} E F=A B, \quad \lim _{x \rightarrow 0} F C=B C,
$$

while the sizes of angles vary as

$$
\begin{aligned}
& \lim _{x \rightarrow 0} \angle A E F=\pi, \\
& \lim _{x \rightarrow 0} \angle E F C=B .
\end{aligned}
$$

By (3.1), there exists some $\delta_{1}$ such that the condition (i) holds for any $x<\delta_{1}$.

By Lemma 2.1, we have

$$
R(E ; T) \subseteq\left\{\{a E, b \beta, c \gamma\}: a \in \mathbb{Z}^{+}, b, c \in \mathbb{N}, \beta, \gamma \in\{F, A, C, D\}\right\} .
$$

By (3.2) and (3.3), there exists some $\delta_{2}<\delta_{1}$ such that for any $x<\delta_{2}$, we have

$$
2 E+\alpha>2 \pi, \quad \forall \alpha \in\{A, F, C, D\} .
$$

Let $x<\delta_{2}$. Then $a=1$ in (3.4). By Lemma 3.1 and the limit (3.2), there exists some $\delta_{3}<\delta_{2}$ such that for any $x<\delta_{3}$, we have

$$
E+b \beta+c \gamma \neq 2 \pi, \quad \forall b, c \in \mathbb{N}, \beta, \gamma \in\{A, C, D\}
$$


Let $x<\delta_{3}$. In view of (3.4), we have

$$
R(E ; T) \subseteq\left\{\{E, b F, c \gamma\}: b \in \mathbb{Z}^{+}, c \in \mathbb{N}, \gamma \in\{A, C, D\}\right\} .
$$

Note that

$$
E+F=\pi+B .
$$

So, every sum- $2 \pi$-collection $\{E, b F, c \gamma\}$, with $b \geq 1$, corresponds to a sum- $(\pi-B)$ collection $\left\{b^{\prime} F, c \gamma\right\}$, where $b^{\prime}, c \in \mathbb{N}$ and $\gamma \in\{A, C, D\}$. By Lemma 3.1 and the limit (3.3), there exists some $\delta_{4}<\delta_{3}$ such that for any $x<\delta_{4}$, we have

$$
b^{\prime} F+c \gamma \neq \pi-B, \quad \forall b^{\prime} \in \mathbb{Z}^{+}, c \in \mathbb{N}, \gamma \in\{A, C, D\} .
$$

Let $x<\delta_{4}$. So $b^{\prime}=0$ and consequently $b=1$. By (3.6), we see that $c \geq 1$. This proves that

$$
R(E ; T) \subseteq\left\{\{E, F, c \gamma\}: c \in \mathbb{Z}^{+}, \gamma \in\{A, C, D\}\right\} .
$$

Along the same lines, we have

$$
R(F ; T) \subseteq\left\{\{a F, b \beta, c \gamma\}: a \in \mathbb{Z}^{+}, b, c \in \mathbb{N}, \beta, \gamma \in\{E, A, C, D\}\right\} .
$$

By Lemma 3.1 and the limit (3.3), there exists some $\delta_{5}<\delta_{4}$ such that for any $x<\delta_{5}$,

$$
a F+b \beta+c \gamma \neq 2 \pi, \quad \forall a \in \mathbb{Z}^{+}, b, c \in \mathbb{N}, \beta, \gamma \in\{A, C, D\} .
$$

In view of (3.8), we deduce that

$$
R(F ; T) \subseteq\left\{\{a F, b E, c \gamma\}: a, b \in \mathbb{Z}^{+}, c \in \mathbb{N}, \gamma \in\{A, C, D\}\right\} .
$$

So any sum- $2 \pi$-collection of $F$ is a sum- $2 \pi$-collection of $E$. From (3.7), we get $R(F ; T)=R(E ; T)$. This proves the condition (ii).

Take $\delta_{E}=\delta_{5}$. By Lemma 3.1, there exists some $\delta_{F}$ depending on the choice of $E$ such that the condition (iii) holds for any $F \in B C$ with $B F<\delta_{F}$. This completes the proof.

We remark that the points $E$ and $F$ can be chosen from any other pair of adjacent edges of $Q$, subject to analogous conditions. This idea will be employed in the proof of Theorem 4.1.

Fix two points $E^{0} \in A B$ and $F^{0} \in B C$ satisfying the conditions (i)-(iii), and consider the resulting pentagon $P^{0}=A^{0} E^{0} F^{0} C^{0} D^{0}$, where $A^{0}=A, C^{0}=C$, and $D^{0}=D$. Now we recursively define a sequence $\left\{P^{k}\right\}_{k \geq 1}$ of proper pentagonal cross-sections.

Let $\pi$ be a plane which crosses $\mathcal{P}$ nontrivially. Let $l$ be a line in $\pi$. For any $\varepsilon>0$, denote by $\pi_{+}^{\varepsilon}$ (resp. $\pi_{-}^{\varepsilon}$ ) the plane obtained by rotating $\pi$ around $l$ by the angle $\varepsilon$ (resp. $-\varepsilon$ ). It is clear that there exists some $\varepsilon$ such that at least one of the planes $\pi_{+}^{\varepsilon}$ and $\pi_{-}^{\varepsilon}$ crosses $\mathcal{P}$ nontrivially. Denote

$$
p(\pi ; l ; \varepsilon)= \begin{cases}\pi_{+}^{\varepsilon}, & \text { if } \pi_{+}^{\varepsilon} \text { crosses } \mathcal{P} \text { nontrivially } \\ \pi_{-}^{\varepsilon}, & \text { otherwise. }\end{cases}
$$


Intuitively, the plane $p(\pi ; l ; \varepsilon)$ is obtained by rotating $\pi$ a little along $l$. For simplifying notation, we use $\operatorname{cr}(\pi ; l ; \varepsilon)$ to denote the intersection $C(p(\pi ; l ; \varepsilon))$. We say that a cross-section is proper if none of its vertices is a vertex of $\mathcal{P}$. Let $\mathcal{C}_{\mathcal{P}}$ be the set of proper pentagonal cross-sections of $\mathcal{P}$. Since every vertex of $Q$ has valence 3 , there exists some $\delta_{0}>0$ such that

$$
\operatorname{cr}\left(P^{0} ; E^{0} F^{0} ; \varepsilon\right) \in \mathcal{C}_{\mathcal{P}}
$$

for any $0<\varepsilon \leq \delta_{0}$. Define

$$
P^{1}=\operatorname{cr}\left(P^{0} ; E^{0} F^{0} ; \delta_{0}\right)=A^{1} E^{1} F^{1} C^{1} D^{1}
$$

to be the initial cross-section. In particular, we have $E^{1}=E^{0}$ and $F^{1}=F^{0}$. Suppose that $P^{k} \in \mathcal{C}_{\mathcal{P}}$ is well defined for some $k \geq 1$. Let $e_{1}^{k}, e_{2}^{k}, \ldots, e_{5}^{k}$ be the edges of $P^{k}$. It is clear that there exists some $0<\delta_{k}<\delta_{k-1}$ such that for any $0<\varepsilon \leq \delta_{k}$ and any edge $e_{j}^{k}$, we have

$$
\operatorname{cr}\left(P^{k} ; e_{j}^{k} ; \varepsilon\right) \in \mathcal{C}_{\mathcal{P}} .
$$

Choosing an edge $e_{j}^{k}$, we can define

$$
P^{k+1}=\operatorname{cr}\left(P^{k} ; e_{j}^{k} ; \delta_{k}\right)
$$

Then $P^{k+1} \in \mathcal{C}_{\mathcal{P}}$. Note that the cross-section $P^{k+1}$ depends on the choices of $e_{j}^{k}$ and $\delta_{k}$, while the value of $\delta_{k}$ depends on $P^{k}$ but is independent of the choice of $e_{j}^{k}$. We call the above procedure of getting $P^{k+1}$ from $P^{k}$ the slight-rotating operation.

Since all cross-sections $P^{k}$ are proper, the slight-rotating operation has a signpreserving property if we take $\delta_{k}$ small enough. Now we clarify the property. Denote by $\operatorname{sgn}(x)$ the signum function, i.e., for any real number $x$,

$$
\operatorname{sgn}(x)= \begin{cases}1, & \text { if } x>0 \\ 0, & \text { if } x=0 \\ -1, & \text { if } x<0\end{cases}
$$

Let $i \geq 0, N>0,1 \leq j \leq 5$ and $a_{1}, \ldots, a_{5}, b_{1}, \ldots, b_{5} \in \mathbb{N}$. Let $V_{i}^{1}, \ldots, V_{i}^{5}$ be the angles of $P^{i}$. Let

$$
\begin{aligned}
& x_{i}=\operatorname{sgn}\left(N-\sum_{l=1}^{5} a_{l} V_{i}^{l}\right), \\
& y_{i}=\operatorname{sgn}\left(e_{j}^{i}-\sum_{l=1}^{5} b_{l} e_{l}^{i}\right) .
\end{aligned}
$$

If $x_{i} y_{i} \neq 0$, then there exists some $0<\delta \leq \delta_{i}$ such that for any $1 \leq j \leq 5$, the crosssection

$$
P^{i+1}=\operatorname{cr}\left(P^{i} ; e_{j}^{i} ; \delta\right)
$$


satisfies $x_{i+1}=x_{i}$ and $y_{i+1}=y_{i}$. It is easy to show the above property if one regards $P^{i+1}$ as a continuous function of the variable $\delta_{i}$ in the definitions (3.9) and (3.10).

With the aid of this property, we deduce that each cross-section in the sequence $\left\{P^{k}\right\}_{k \geq 1}$ satisfies some conditions analogous to (i)-(iii) as if the $\delta_{i}$ 's are chosen to be small enough. For all $k \geq 2$, we name the vertices of the pentagon $P^{k}$ by

$$
P^{k}=A^{k} E^{k} F^{k} C^{k} D^{k}
$$

in the natural way that $E^{k} \in A B$ and $F^{k} \in B C$.

Theorem 3.3 For any $k \geq 1$, there exists some $\delta_{k-1}^{*} \leq \delta_{k-1}$ such that for any edge $e_{j_{1}}^{1}$ of $P^{1}$, any edge $e_{j_{2}}^{2}$ of $P^{2}, \ldots$, and any edge $e_{j_{k-1}}^{k-1}$ of $P^{k-1}$, the cross-section $P^{k}$ defined by (3.10) satisfies

(i') $2 A^{k} E^{k}<\min \left\{E^{k} F^{k}, F^{k} C^{k}, C^{k} D^{k}, D^{k} A^{k}\right\}$;

(ii') $R\left(E^{k} ; P^{k}\right)=R\left(F^{k} ; P^{k}\right) \subseteq\left\{\left\{E^{k}, F^{k}, c \gamma\right\}: c \in \mathbb{Z}^{+}, \gamma \in\left\{A^{k}, C^{k}, D^{k}\right\}\right\}$;

(iii') for any $a, b \in \mathbb{N}, c \in\{0, \pm 1, \pm 2\}$ and $l \in\left\{E^{k} F^{k}, 2 F^{k} C^{k}\right\}$, we have

$$
l \neq a \cdot C^{k} D^{k}+b \cdot D^{k} A^{k}+c \cdot A^{k} E^{k}
$$

(iv') if there exist some $a \in \mathbb{Z}^{+}, b, c \in \mathbb{N}, N \in\{\pi, 2 \pi\}$, and three pairwise distinct angles $V_{k}^{j_{1}}, V_{k}^{j_{2}}, V_{k}^{j_{3}}$ of $P^{k}$ such that

$$
N=a V_{k}^{j_{1}}+b V_{k}^{j_{2}}+c V_{k}^{j_{3}}
$$

then for any $0 \leq h \leq k$, the corresponding angles $V_{h}^{j_{1}}, V_{h}^{j_{2}}, V_{h}^{j_{3}}$ of $P^{h}$ satisfy

$$
N=a V_{h}^{j_{1}}+b V_{h}^{j_{2}}+c V_{h}^{j_{3}}
$$

Let $\delta_{i}^{*}(i \geq 0)$ be defined as in the above theorem. The next lemma will be used to exclude some cases from the set of $2 \pi$-sum-collections of the specified angle $E^{k+1}$.

Lemma 3.4 Let $\mathcal{P} \in \mathcal{E}$ and $k \in \mathbb{Z}^{+}$. Suppose that

$$
\left\{E^{k}, F^{k}, A^{k}\right\} \in R\left(E^{k} ; P^{k}\right) .
$$

Then we have

$$
\left\{E^{k+1}, F^{k+1}, A^{k+1}\right\} \notin R\left(E^{k+1} ; \operatorname{cr}\left(P^{k} ; C^{k} D^{k} ; \delta_{k}^{*}\right)\right) .
$$

Similarly, if

$$
\left\{E^{k}, F^{k}, C^{k}\right\} \in R\left(E^{k} ; P^{k}\right)
$$

then we have

$$
\left\{E^{k+1}, F^{k+1}, C^{k+1}\right\} \notin R\left(E^{k+1} ; \operatorname{cr}\left(P^{k} ; D^{k} A^{k} ; \delta_{k}^{*}\right)\right) .
$$


Fig. 4 The parallel relation $F^{k} F^{k+1} C^{k} \| D^{k} A^{k} A^{k+1}$

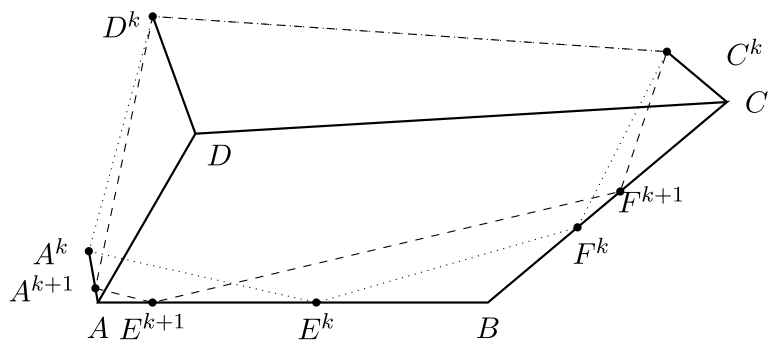

Proof By (3.11), we have

$$
F^{k} C^{k} \| D^{k} A^{k}
$$

Denote $\operatorname{cr}\left(P^{k} ; C^{k} D^{k} ; \delta_{k}^{*}\right)=A^{k+1} E^{k+1} F^{k+1} C^{k} D^{k}$. If (3.12) is false, then we have

$$
F^{k+1} C^{k} \| D^{k} A^{k+1}
$$

It is clear that the points $F^{k}$ and $F^{k+1}$ are distinct, while the points $A^{k}$ and $A^{k+1}$ are also distinct. Hence by (3.14) and (3.15), we find parallel facets $F^{k} F^{k+1} C^{k} \|$ $D^{k} A^{k} A^{k+1}$ (see Fig. 4). But $\mathcal{P} \in \mathcal{E}$, thus we obtain a contradiction. The relation (3.13) can be proved similarly. This completes the proof.

\section{The Main Result}

In this section, we complete the proof of Theorem 1.2.

Let $k \in \mathbb{Z}^{+}$. Suppose that $\mathcal{T}_{k}$ is a tessellation of the plane by copies of $P^{k}$. Denote the copies used in $\mathcal{T}_{k}$ by

$$
\left\{P_{i}^{k}=A_{i}^{k} E_{i}^{k} F_{i}^{k} C_{i}^{k} D_{i}^{k}: i \in \Lambda_{k}\right\},
$$

where $\Lambda_{k}$ is a set. Note that each copy in $\mathcal{T}^{k}$ is arranged counter-clockwise either in the order

$$
A_{i}^{k}, E_{i}^{k}, F_{i}^{k}, C_{i}^{k}, D_{i}^{k}
$$

or in the order

$$
A_{j}^{k}, D_{j}^{k}, C_{j}^{k}, F_{j}^{k}, E_{j}^{k} .
$$

Denote by $\mathcal{I}_{k}$ the set of indices $i \in \Lambda_{k}$ such that the vertices of $P_{i}^{k}$ are arranged counter-clockwise in the order (4.1). Without loss of generality, we can always suppose that $1 \in \mathcal{I}_{k}$.

A quadrilateral is said to be cyclic if all its vertices lie on the same circle. Recall that $Q$ is a quadrilateral facet of $\mathcal{P}$.

Theorem 4.1 The facet $Q$ is either a parallelogram or a cyclic quadrilateral. 

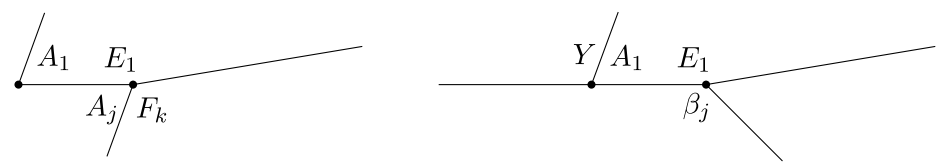

Fig. 5 The local tessellations of $A_{1}$ and $E_{1}$

Proof Assume, to the contrary, that $Q$ is neither a parallelogram nor cyclic. Without loss of generality, we can suppose that $C$ is one of the largest angles of $Q$. Consider the cross-section

$$
P^{1}=\operatorname{cr}\left(P^{0} ; E^{0} F^{0} ; \delta_{1}^{*}\right) .
$$

For convenience, we rewrite the copies of $P^{1}$ as $P_{i}^{1}=A_{i} E_{i} F_{i} C_{i} D_{i}$. Since $P^{1}$ is a tiler, we can express $S\left(E_{1}\right)=\left[E_{1}, \beta_{j}, X\right]$, where $\beta_{j} \in\left\{A_{j}, E_{j}, F_{j}, C_{j}, D_{j}\right\}$, and $X$ is a sequence of angles. By the condition (ii'), we have $\beta_{j} \neq E_{j}$.

Assume that $\beta_{j}=A_{j}$. By (i'), the sequence $X$ contains no angle $A_{i}$. By (ii'), we deduce that $S\left(E_{1}\right)=\left[E_{1}, A_{j}, F_{k}\right]$ for some $k \in \Lambda_{1}$ (see Fig. 5). It follows that $E^{1}+$ $A^{1}+F^{1}=2 \pi$. By the condition (iv'), we obtain $C+D=\pi$. So $Q$ is a parallelogram, which is a contradiction.

Below we can suppose that $\beta_{j} \in\left\{F_{j}, C_{j}, D_{j}\right\}$. In this case, the condition (i') implies $S\left(A_{1}\right)=\left[A_{1}, Y, \pi\right]$, where $Y$ is a sequence of angles (see Fig. 5). By (i') and (ii'), we deduce that $Y$ contains no angle $A_{i}$. Thus there exist some $b \in \mathbb{Z}^{+}$ and $\beta^{1} \in\left\{C^{1}, D^{1}\right\}$ such that $A^{1}+b \beta^{1}=\pi$. By (iv'), we find that $A+b \beta=\pi$ for some $\beta \in\{C, D\}$. Since $Q$ is neither a parallelogram nor cyclic, we see that $b \geq 2$. But $C$ is one of the largest angles, so $\beta=D$. Namely,

$$
A+b D=\pi
$$

Consider $E^{\prime} \in A D$ and $F^{\prime} \in C D$ subject to the conditions corresponding to (i')-(iv'). Since $C$ is one of the largest angles, we derive

$$
A+b^{\prime} B=\pi
$$

for some $b^{\prime} \geq 2$. Adding (4.2) and (4.3) yields

$$
2 \pi=2 A+b D+b^{\prime} B \geq 2(A+B+D)=2(2 \pi-C),
$$

namely $C \geq \pi$, hence we obtain a contradiction. This completes the proof.

Theorem 4.2 The facet $Q$ is cyclic.

Proof Assume to the contrary that $Q$ is a non-cyclic facet. By Theorem 4.1, it is a parallelogram. Without loss of generality, we can suppose that $C$ is one of the smallest angles of $Q$, and that $A+D=\pi$. Since $Q$ is non-cyclic, we deduce that the angles $C$ and $D$ have distinct sizes. Therefore, the condition (ii') implies that

$$
R\left(E^{0} ; P^{0}\right) \subseteq\left\{\left\{E^{0}, F^{0}, A^{0}\right\},\left\{E^{0}, F^{0}, C^{0}\right\}\right\} .
$$


Fig. 6 The cross-sections $P^{1}$ (dotted), $P^{2}$ (dashed) and $P^{3}$ (thin)

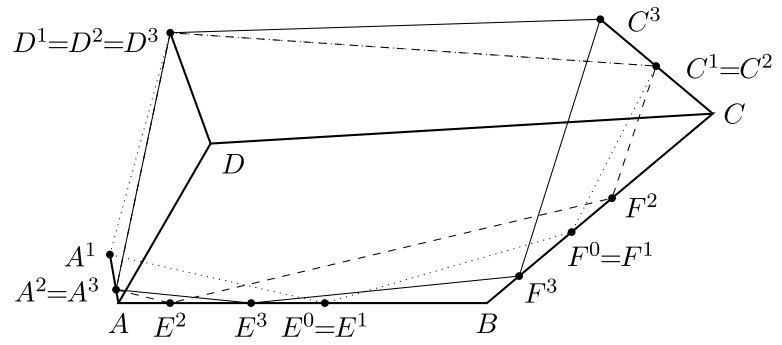

Consider the cross-sections (see Fig. 6)

$$
\begin{aligned}
& P^{1}=\operatorname{cr}\left(P^{0} ; E^{0} F^{0} ; \delta_{2}^{*}\right)=A^{1} E^{1} F^{1} C^{1} D^{1}, \\
& P^{2}=\operatorname{cr}\left(P^{1} ; C^{1} D^{1} ; \delta_{2}^{*}\right)=A^{2} E^{2} F^{2} C^{2} D^{2}, \\
& P^{3}=\operatorname{cr}\left(P^{2} ; D^{2} A^{2} ; \delta_{2}^{*}\right)=A^{3} E^{3} F^{3} C^{3} D^{3} .
\end{aligned}
$$

By Lemma 3.4 and (iv'), we see that $R\left(E^{3} ; P^{3}\right)=\varnothing$. But the cross-section $P^{3}$ tiles the plane, hence we obtain a contradiction. This completes the proof.

To proceed further, we need the following technical lemma.

\section{Lemma 4.3 Let $k \geq 1$. Suppose that}

$$
R\left(E^{k} ; P^{k}\right)=R\left(F^{k} ; P^{k}\right)=\left\{\left\{E^{k}, F^{k}, D^{k}\right\}\right\} .
$$

Then any edge $F_{i}^{k} C_{i}^{k}$ is not represented. Moreover, any line segment $F_{i}^{k} C_{i}^{k}+C_{j}^{k} F_{j}^{k}$ or $E_{i}^{k} A_{i}^{k}+C_{j}^{k} F_{j}^{k}$ (if it exists) is not represented.

Proof For convenience, rewrite the copies $\left\{P_{i}^{k}: i \in \Lambda_{k}\right\}$ as $P_{i}^{k}=A_{i} E_{i} F_{i} C_{i} D_{i}$. Assume to the contrary that $F_{i} C_{i}$ is represented. By (4.7), there is no point $F_{j}(j \neq i)$ lying on the edge $F_{i} C_{i}$, and there is at most one point $E_{j}$ lying on $F_{i} C_{i}$. Therefore, we have

$$
F^{k} C^{k}=a_{1} \cdot C^{k} D^{k}+b_{1} \cdot D^{k} A^{k}+c_{1} \cdot A^{k} E^{k}
$$

for some $a_{1}, b_{1} \in \mathbb{N}$ and $c_{1} \in\{0,1\}$. This contradicts the condition (iii'). Hence $F_{i} C_{i}$ is not represented. For the same reason, any line segment $F_{i} C_{i}+C_{j} F_{j}$ (if it exists) is not represented.

Suppose that $1 \in \mathcal{I}_{k}$ and the line segment $E_{1} A_{1}+C_{2} F_{2}$ is represented in $\mathcal{T}_{k}$. Then $2 \in \mathcal{I}_{k}$ (see Fig. 7). We claim that there exist some $i, j \in \Lambda_{k}$ such that

$$
S\left(E_{1}\right)=\left[E_{1}, F_{i}, D_{j}\right]
$$

In fact, by (4.7), there is at most one copy $P_{i}^{k}$ such that the vertex $E_{i}$ lying on the line segment $E_{1} F_{2}$, where $i \neq 1,2$. Also, there is at most one point $F_{j}(j \neq 1,2)$ lying on the line segment $E_{1} F_{2}$. If the sequence $S\left(E_{1} F_{2}\right)$ contains an edge $E_{i} F_{i}$, then the 
Fig. 7 The tessellation of the $\varepsilon$-neighborhood of the line segment $E_{1} F_{2}$

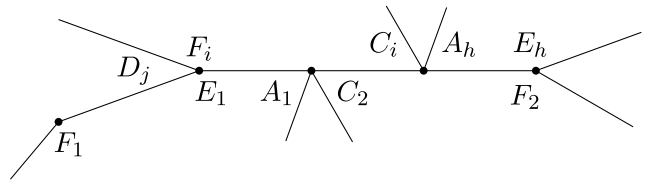

condition (ii') implies (4.8). Otherwise, by (iii'), we can deduce that $S\left(E_{1} F_{2}\right)$ contains an edge $F_{i} C_{i}$. Thus, the condition (i') yields $S\left(E_{1} A_{1}, C_{2} F_{2}\right)=\left[F_{i} C_{i}, A_{h} E_{h}\right]$ for some $i$ and $h$. In particular, the relation (4.8) holds. The proves the claim.

By (4.8), the edge $E_{1} F_{1}$ is represented. It follows that there is at most one point $E_{i}$ $(i \neq 1)$ lying on the edge $E_{1} F_{1}$, and there is no point $F_{j}(j \neq 1)$ lying on $E_{1} F_{1}$. So there exist some $a_{2}, b_{2} \in \mathbb{N}$ and $c_{2} \in\{0,1\}$ such that

$$
E^{k} F^{k}=a_{2} \cdot C^{k} D^{k}+b_{2} \cdot D^{k} A^{k}+c_{2} \cdot A^{k} E^{k},
$$

contradicting the condition (iii'). This completes the proof.

Now we are in a position to complete the proof of the main result.

Proof of Theorem 1.2 It suffices to show that $\varepsilon=\varnothing$. Assume, to the contrary, that $\mathcal{P} \in \mathcal{E}$. By Theorem 4.2, we see that $Q$ is cyclic. Suppose that $D$ is one of the smallest angles of $Q$. By the condition (ii'), we have

$$
R\left(E^{0} ; P^{0}\right) \subseteq\left\{\left\{E^{0}, F^{0}, A^{0}\right\},\left\{E^{0}, F^{0}, C^{0}\right\},\left\{E^{0}, F^{0}, D^{0}\right\}\right\} .
$$

Consider the cross-sections $P^{1}, P^{2}, P^{3}$ defined by (4.4)-(4.6). By (4.9), Lemma 3.4 and the condition (iv' $)$, we see that $R\left(E^{3} ; P^{3}\right) \subseteq\left\{\left\{E^{3}, F^{3}, D^{3}\right\}\right\}$. Since $P^{3}$ is a tiler, by (ii'), we have

$$
R\left(E^{3} ; P^{3}\right)=R\left(F^{3} ; P^{3}\right)=\left\{\left\{E^{3}, F^{3}, D^{3}\right\}\right\} .
$$

By (4.10) and (iv'), we deduce that

$$
R_{\pi}\left(A^{3} ; P^{3}\right) \subseteq\left\{\left\{A^{3}, C^{3}\right\}\right\} \cup\left\{\left\{b A^{3}, a D^{3}\right\}: b \in \mathbb{Z}^{+}, a \in \mathbb{N}\right\} .
$$

Consider the tessellations of the plane by copies of $P^{3}$. For convenience, rewrite

$$
\mathcal{T}=\mathcal{T}_{3}, \quad P_{i}^{3}=A_{i} E_{i} F_{i} C_{i} D_{i}, \quad \Lambda_{3}=\Lambda, \quad \mathcal{I}_{3}=\mathcal{I} .
$$

By (4.10) and (i'), there exist an $m \in \mathbb{Z}^{+}$and some $i_{1}, i_{2}, \ldots, i_{m} \in \Lambda_{3}$ such that

$$
S\left(A_{1}\right)=\left[A_{1}, \alpha_{i_{1}}, \alpha_{i_{2}}, \ldots, \alpha_{i_{m}}, \pi\right],
$$

where $\alpha_{i_{j}} \in\left\{A_{i_{j}}, C_{i_{j}}, D_{i_{j}}\right\}$. Let $\varepsilon>0$. Considering the tessellation of the $\varepsilon$ neighborhood of the point $E_{i_{j}}$, we see that

$$
\alpha_{i_{j}} \neq A_{i_{j}}, \quad \forall 1 \leq j \leq m .
$$

Set $i_{1}=2$. Assume that $m=1$. If $\alpha_{2}=D_{2}$, then $\left\{E^{3}, F^{3}, C^{3}\right\} \in R\left(E^{3} ; P^{3}\right)$, contradicting (4.10). Therefore, by (4.13), the expression (4.12) reduces to $S\left(A_{1}\right)=$ 
Fig. 8 The tessellation for the case of $m=1$

Fig. 9 The tessellation for the case of $S\left(D_{2} C_{2}\right)=\left[D_{3} A_{3}\right]$
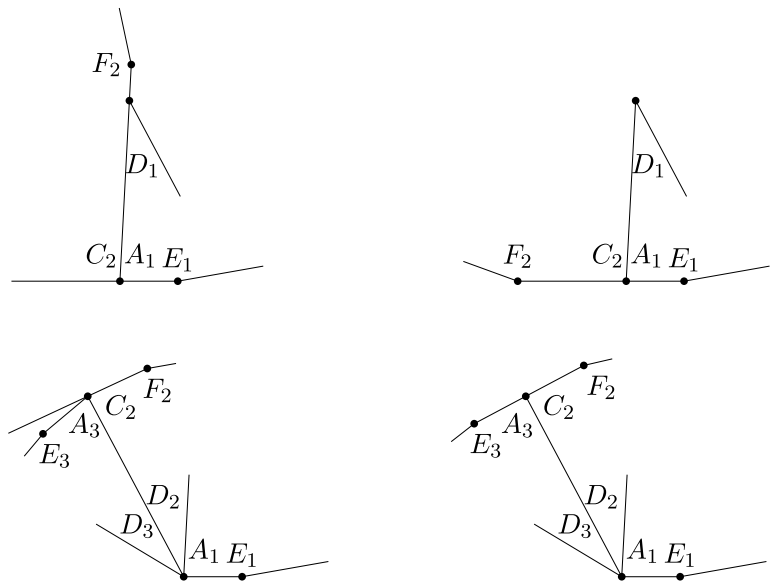

$\left[A_{1}, C_{2}, \pi\right]$ (see Fig. 8). In this case, if $2 \notin \mathcal{I}$, then the point $F_{2}$ lies on the line determined by $D_{1} A_{1}$. By (4.10), we find that the edge $F_{2} C_{2}$ is represented, contradicting Lemma 4.3. So $2 \in \mathcal{I}$. By (4.10), the line segment $E_{1} A_{1}+C_{2} F_{2}$ is represented, also contradicting Lemma 4.3.

Below we can suppose that $m \geq 2$. Set $i_{2}=3$. Then the expression (4.12) reduces to

$$
S\left(A_{1}\right)=\left[A_{1}, D_{2}, D_{3}, \ldots, \pi\right] .
$$

Assume that $2 \in \mathcal{I}$. By Lemma 4.3, the edge $F_{2} C_{2}$ is not represented. So there exist an $s \in \mathbb{Z}^{+}$and some $j_{1}, j_{2} \ldots, j_{s} \in \Lambda_{3}$ such that

$$
S\left(C_{2}\right)=\left[C_{2}, \pi, \beta_{j_{1}}, \beta_{j_{2}}, \ldots, \beta_{j_{s}}\right],
$$

where $\beta_{i}$ is an angle of the copy $T_{i}$. Therefore the edge $D_{2} C_{2}$ is represented. In view of (4.10), (4.14), and (4.15), the line segment $D_{2} C_{2}$ contains neither a point $E_{i}$, nor a point $F_{i}$, for some $i$. Moreover, by (4.10), no point $A_{j}$ lies in the interior of $D_{2} C_{2}$, since otherwise the $\varepsilon$-neighborhood of the point $E_{j}$ cannot be tiled. For the same reason, there is no point, say, $C_{j}$, lying in the interior of $D_{2} C_{2}$. Therefore,

$$
S\left(D_{2} C_{2}\right) \in\left\{\left[D_{3} A_{3}\right],\left[D_{3} C_{3}\right]\right\} .
$$

So $j_{s}=3$. If $S\left(D_{2} C_{2}\right)=\left[D_{3} A_{3}\right]$, then either the edge $A_{3} E_{3}$ is represented (when $s \geq 2$ ), which is impossible by the conditions $\left(\mathrm{i}^{\prime}\right)$ and (4.10); or the line segment $E_{3} A_{3}+C_{2} F_{2}$ is represented (when $s=1$ ), which contradicts Lemma 4.3. See Fig. 9. In the other case, $S\left(D_{2} C_{2}\right)=\left[D_{3} C_{3}\right]$ (see Fig. 10). Then either $F_{3} C_{3}$ is represented (when $s \geq 2$ ), or $F_{2} C_{2}+C_{3} F_{3}$ is represented (when $s=1$ ). Both of them contradict Lemma 4.3.

Hence we have $2 \notin \mathcal{I}$. Considering the tessellation of the $\varepsilon$-neighborhood of the point $E_{2}$, we find that

$$
S\left(A_{2}\right)=\left[A_{2}, \pi, \gamma_{h_{1}}, \gamma_{h_{2}}, \ldots, \gamma_{h_{t}}\right]
$$


Fig. 10 The tessellation for the case of $S\left(D_{2} C_{2}\right)=\left[D_{3} C_{3}\right]$
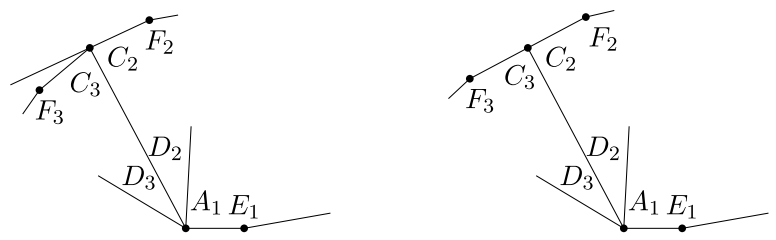

Fig. 11 The tessellation for the case of $S\left(D_{2} A_{2}\right)=\left[D_{3} A_{3}\right]$
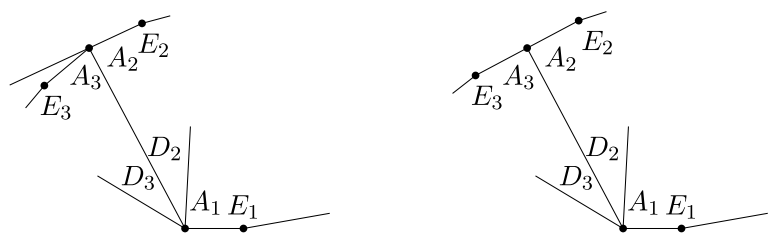

Fig. 12 The tessellation for the case of $S\left(D_{2} A_{2}\right)=\left[D_{3} C_{3}\right]$
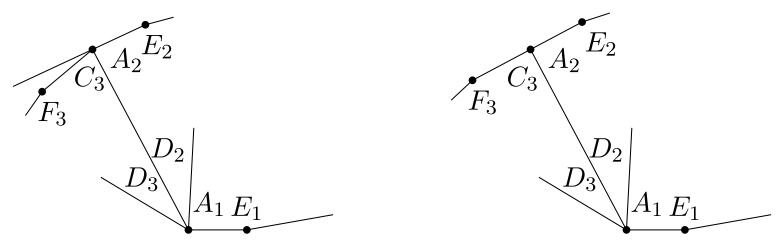

Fig. 13 Three classes of universal tilers
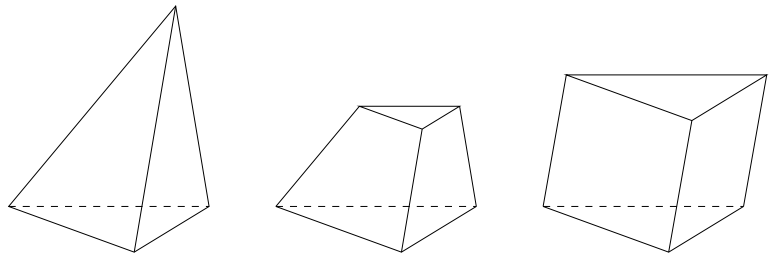

where $t \geq 1$ and $\gamma_{h_{i}}$ is an angle of the copy $T_{h_{i}}$. Therefore $D_{2} A_{2}$ is represented. For the same reason as in the case of $2 \in \mathcal{I}$, we deduce that

$$
S\left(D_{2} A_{2}\right) \in\left\{\left[D_{3} A_{3}\right],\left[D_{3} C_{3}\right]\right\} .
$$

If $S\left(D_{2} A_{2}\right)=\left[D_{3} A_{3}\right]$, then either $A_{3} E_{3}$ is represented (when $t \geq 2$ ), or $E_{2} A_{2}+$ $A_{3} E_{3}$ is represented (when $t=1$ ). See Fig. 11 . Both of them are absurd by the condition (i'). In the other case $S\left(D_{2} A_{2}\right)=\left[D_{3} C_{3}\right]$. Then either $C_{3} F_{3}$ is represented (when $t \geq 2$ ), or $F_{3} C_{3}+A_{2} E_{2}$ is represented. See Fig. 12. Both of them contradict Lemma 4.3.

To sum up, the cross-section $P^{3}$ does not tile the plane. This implies that $\mathcal{E}=\varnothing$, and that completes the proof.

We conclude this paper by giving a graphical illustration of our main result. Let $P$ be a pentahedronal universal tiler. Since any quadrilateral-based pyramid has no parallel facets, we see that $P$ has two triangular bases. Moreover, the parallel facets of $P$ must be its two triangular bases. It turns out that either all quadrilateral facets of $P$ are trapezoids, or $P$ is a triangular prism. In the former case, the pentahedron $P$ can be obtained from cutting a tetrahedron by a plane parallel to one of its facets. In conclusion, universal tilers have three classes as illustrated in Fig. 13. 
Acknowledgements This work was supported by the China Postdoctoral Science Foundation (Grant No. 20110490224), and in part by the National Natural Science Foundation of China (Grant No. 11101010). I am indebted to Jin Akiyama for his constant encouragement. I am grateful to Rongquan Feng, Gopala Krishna, János Pach, Liping Yuan, and Chuanming Zong for their comments and suggestions, which were helpful in the preparation of the present revised form of this paper.

\section{References}

1. Akiyama, J.: Tile-makers and semi-tile-makers. Am. Math. Mon. 114, 602-609 (2007)

2. Brass, P., Moser, W.O.J., Pach, J.: Research Problems in Discrete Geometry. Springer, Berlin (2005)

3. Grünbaum, B., Shephard, G.C.: Tilings and Patterns. Freeman, New York (1986)

4. Hirschhorn, M.D., Hunt, D.C.: Equilateral convex pentagons which tile the plane. J. Comb. Theory, Ser. A 39, 1-18 (1985)

5. Reinhardt, K.: Über die Zerlegung der Ebene in Polygone, Dissertation, Universität Frankfurt (1918)

6. Sugimoto, T., Ogawa, T.: Properties of tilings by convex pentagons. Forma 21, 113-128 (2006)

7. Wang, D.G.L.: On universal tilers. arXiv:1109.0813 [math.CO] 